\title{
Importancia de la detección del trastorno dismórfico corporal en la consulta de Girugía Estética
}

\section{The importance of diagnosing body dysmorphic disorder in the Aesthetic Surgery consultation}

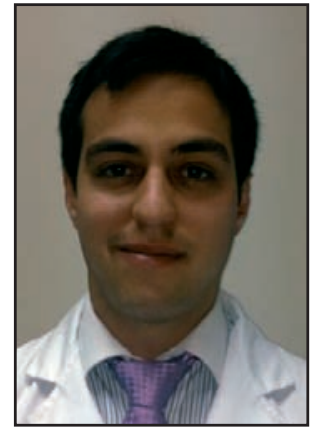

Rochefort-Ciscutti, G.

\author{
Rochefort-Ciscutti, G.*, Berner-Gómez, J.E.*, \\ Castillo-Montoya, P.**, Vidal-García-Huidobro, P. **
}

\section{Resumen}

El auge de la Cirugía Estética ha motivado que cobre mayor interés el estudiar las motivaciones de los pacientes para acudir a este tipo de consultas. En el denominado Trastorno Dismórfico Corporal (TDC), los pacientes sufren por la percepción de un defecto físico imaginario. Se ha generado controversia entre los especialistas, sobre el eventual perjuicio que tendría operar a este tipo de pacientes considerando las posibles implicaciones médico-legales.

Nos planteamos como objetivo el realizar una revisión sistemática de la literatura para caracterizar epidemiológicamente el TDC en el contexto de la consulta de Cirugía Estética, considerando su prevalencia, comorbilidades y las consecuencias de los procedimientos quirúrgicos en la salud mental de este tipo de pacientes.

Diseñamos una búsqueda sistemática en MEDLINE utilizando los términos MESH: "Body Dysmorphic Disorders" y "Surgery, Plastic".

Encontramos 14 artículos, todos en inglés a los que agregamos otras 21 publicaciones relevantes. La definición de TDC más ampliamente difundida es la del DSM-IV TR. La prevalencia es significativamente mayor entre consultantes por Cirugía Estética (6-15\%) que entre la población general $(2,4 \%)$. Las comorbilidades más frecuentes son los trastornos depresivos, la ansiedad y trastornos del control de impulsos. Los pacientes suelen presentar pensamientos intrusivos e ideas sobrevaloradas de su imagen corporal asociados a egodistonía con escaso nivel de conciencia de la enfermedad (insight), que les lleva a padecer una mayor incidencia de intentos suicidas. Presentan además un bajo nivel de satisfacción postquirúrgica.

Consideramos que es fundamental la detección del TDC en la consulta de Cirugía Estética para una derivación oportuna de estos pacientes hacia un tratamiento adecuado. Ampliar los estudios en nuestra región permitirá comparar nuestra situación local con lo publicados sobre otras poblaciones y validar así instrumentos de cribado de estos pacientes.
Palabras clave Cirugía Estética,

Síndrome dísmórfico corporal, Selección de pacientes.
III

\begin{abstract}
Along with the boom in Cosmetic Surgery, interest has increased in understanding the patients' motivations for attending an Aesthetic Surgery consultation. Patients suffering from Body Dysmorphic Disorder (BDD) perceive imaginary body defects, producing discomfort. Controversy has risen among experts about the possible negative outcomes that Aesthetic Surgery could have in them.

Our objective is to conduct a systematic review of the published literature about BDD and Aesthetic Surgery, considering prevalence, comorbidities and consequences of surgical procedures in their mental health.

We designed a MEDLINE search strategy using appropriate MeSH terms: "Body Dysmorphic Disorders" and "Surgery, Plastic".

The most widely used definition is the DSM-IV TR. BDD prevalence is significantly higher among Aesthetic Surgery patients (6$15 \%$ ) than in the general population $(2.4 \%)$. Most common comorbidities are: depressive disorders, anxiety and impulse control disorders. These patients usually present intrusive egodistonic thoughts and overvalued body image ideas, associated with poor insight,which leads to higher incidence of suicide attempts. Studies have shown low postoperative satisfaction among them.

We believe that early BDD detection is critical for aesthetic surgeons, in order to properly refer and treat these patients. There is scarce research about the subject in our region, which is necessary to compare the phenomenon in our setting with the published literature abroad.
\end{abstract}

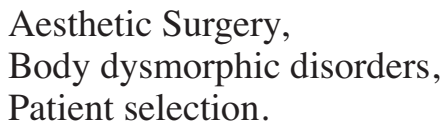

Aesthetic Surgery,

Body dysmorphic disorders, Patient selection.

Level of evidence $\quad$ III 


\section{Introducción}

Con el explosivo crecimiento de la Cirugía Estética en las últimas décadas, han cobrado mayor importancia las comorbilidades psiquiátricas de los pacientes que consultan en esta especialidad. Se trata de un grupo diverso y con distintas motivaciones, destacando por su hostilidad aquellos que, en el contexto de su descompensación, amenazan física y legalmente a sus cirujanos. El grado extremo de esta situación son los 5 casos publicados de homicidio de cirujanos por parte de pacientes que no quedaron conformes con sus resultados postoperatorios $(1,2)$.

El Trastorno Dismórfico Corporal (TDC) es una entidad psiquiátrica clasificada dentro de los trastornos somatomorfos (3); fue descrito por primera vez en 1891 por el italiano Enrico Morselli, quien acuñó el término dismorfofobia (4). Como muchos otros trastornos psiquiátricos, pasaron décadas hasta que sus criterios diagnósticos quedaran estandarizados y no fue hasta 1987 cuando la Asociación Psiquiátrica Americana incorporó esta entidad en el DSM-III-R. Desde entonces el término dismorfofobia ha entrado en desuso, siendo reemplazado por el de Trastorno Dismórfico Corporal (5).

En los criterios diagnósticos de la última versión del DSM-IV-TR (3) destaca la excesiva preocupación de los pacientes por algún defecto imaginario de su aspecto físico. Las personas que sufren TDC suelen intentar corregir las anomalías que perciben ocultándolas de maneras ingeniosas e incluso recurriendo a la Cirugía Estética.

Este fenómeno ha producido una extensa discusión entre cirujanos plásticos que sostienen que los pacientes con TDC no se verían beneficiados por los procedimientos quirúrgicos propios de esta especialidad, ya que los defectos que ellos perciben no se van a modificar dada su naturaleza imaginaria (2). Esto, sumado a las consecuencias médico-legales que conlleva operar a estos pacientes, ha movido a caracterizar a los pacientes para detectar quiénes deben ser excluidos de una cirugía estética.

La presente revisión sistemática de la literatura pretende caracterizar epidemiológicamente el TDC en el contexto de la consulta de Cirugía Estética, considerando su prevalencia, comorbilidades, características generales y los efectos de los procedimientos quirúrgicos en la salud mental de estos pacientes.

\section{Material y método}

Diseñamos una búsqueda en MEDLINE utilizando los términos MESH idóneos: ("Body Dysmorphic Disorders" AND "Surgery, Plastic"). Definimos previamente que solo serían revisados artículos que relacionaran el TDC con la Cirugía Estética, que estuvieran publicados en español o inglés, excluyendo trabajos exclusivamente reconstructivos, traumatológicos o de otras disciplinas. Los artículos fueron revisados por 2 autores de forma paralela, teniendo la potestad en consenso de proponer bibliografía complementaria relevante que fuera citada por el resto de los artículos encontrados.

\section{RESULTADOS}

Encontramos 14 artículos, todos en inglés (Tabla I) (2, 6-18). Los revisores consideraron todos ellos relevantes y posteriormente, agregaron otras 21 publicaciones citadas reiteradamente en la literatura encontrada.

\section{Definición y Prevalencia}

La definición del Trastorno Dismórfico Corporal más ampliamente utilizada es la ya mencionada del DSM-IVTR (3) (Tabla II).Ocasionalmente, a modo de referencia, se menciona su clasificación en el CIE-10 dentro del grupo de los trastornos hipocondríacos (19).

Dado que próximamente se publicará la quinta versión del DSM, cabe mencionar que los criterios diagnósticos de TDC no tendrán mayores cambios (Tabla III) (20). Resulta novedoso el hecho de que se piensa incluir el nivel de conciencia de enfermedad o insight en el contexto sociocultural, especificando si es bueno, malo o si llega a niveles delirantes en cuanto al contenido del TDC.

Se han realizado varios estudios de prevalencia en distintas poblaciones. Sostienen que en Estados Unidos la prevalencia en la población general es de 2,4\% (21), recogiéndose de series previas una prevalencia de entre el 0,7\% al $13 \%(22,23)$. A su vez, en Brasil, la prevalencia es del $12 \%$ en población general y en Argentina, en población femenina adolescente, alcanza el $47 \%$ (24). En todos estos estudios se utilizaron distintos instrumentos diagnósticos, lo que dificulta la comparación de resultados.

La prevalencia del TDC en la consulta de Cirugía Estética varía entre el $6 \%$ y el 15\% según Phillips y col. (25). Otra serie, recogida por Ishigookay y col. muestra una prevalencia del 47,7\% (26); en Europa en cambio, se señala que la prevalencia en la consulta de la especialidad sería del 9,1\% (27). A su vez, una investigación realizada por Veale recoge que de una muestra de 50 pacientes diagnosticados de TDC, el 26\% se había sometido a intervenciones estéticas previamente (5). En la consulta dermatológica la prevalencia también sería mayor que en la población general, alcanzando entre un 9,0 y un $12,0 \%$ (25).

\section{Diferencia de género}

Existen 2 artículos en los que se compara la prevalencia por sexos no estableciéndose diferencias significativas; pese a que culturalmente, y con cierto prejuicio, tradicionalmente se ha asociado mayor incidencia del TDC en el sexo femenino (21-28).

\section{Conciencia de enfermedad (insight)}

Es la capacidad de hacer consciente el estado patológico de uno mismo. Algunas enfermedades psiquiátricas cursan con pérdida de conciencia de enfermedad. Un $35,6 \%$ de los pacientes que padecen TDC también tienen 
Tabla I. Artículos encontrados en búsqueda sistemática en MEDLINE

\begin{tabular}{|c|c|c|c|}
\hline Primer autor & $\begin{array}{l}\text { Fecha de } \\
\text { publicación }\end{array}$ & Título & Revista (volumen y páginas) \\
\hline Veale D. ${ }^{7}$ & Marzo 2013 & $\begin{array}{l}\text { Validation of genital appearance } \\
\text { satisfaction scale and the cosmetic } \\
\text { procedure screening scale for women } \\
\text { seeking labiaplasty }\end{array}$ & $\begin{array}{l}\text { Journal of Psychosomatic Obstetrics } \\
\text { and Gynaecology; 34(1):46-52 }\end{array}$ \\
\hline Gupta M.A. ${ }^{8}$ & Enero 2013 & $\begin{array}{l}\text { Evaluation of cutaneous body image } \\
\text { dissatisfaction in the dermatology patient }\end{array}$ & Clinics in Dermatology; 31(1):72-79 \\
\hline Morselli P.G. ${ }^{9}$ & Octubre 2012 & $\begin{array}{l}\text { Should plastic surgeons operate on patients } \\
\text { diagnosed with body dysmorphic disorders? }\end{array}$ & $\begin{array}{l}\text { Plastic and Reconstructive Surgery; } \\
\text { 130(4):620e-622e }\end{array}$ \\
\hline Ericksen W.L. ${ }^{10}$ & Septiembre 2012 & Psychiatric issues in cosmetic plastic surgery & The Psychiatric Quarterly; 83(3):343-352 \\
\hline Kyle A. ${ }^{11}$ & Julio 2012 & Body dysmorphia and plastic surgery & Plastic Surgery Nursing; 32(3):96-98 \\
\hline Clarke A. ${ }^{12}$ & Julio 2012 & $\begin{array}{l}\text { Low priority? A cross sectional study of } \\
\text { appearance anxiety in } 500 \text { consecutive } \\
\text { referrals for cosmetic surgery }\end{array}$ & $\begin{array}{l}\text { Psychology, Health and Medicine; } \\
\text { 17(4):440-446 }\end{array}$ \\
\hline Mulkens $S .{ }^{13}$ & Marzo 2012 & $\begin{array}{l}\text { Psychopathology symptoms in a sample of } \\
\text { female cosmetic surgery patients }\end{array}$ & $\begin{array}{l}\text { Journal of Plastic Reconstructive and } \\
\text { Aesthetic Surgery; 65(3):321-327 }\end{array}$ \\
\hline De Brito M.J. ${ }^{14}$ & Febrero 2012 & $\begin{array}{l}\text { Should Plastic surgeons operate on } \\
\text { patients diagnosed with body dysmorphic } \\
\text { disorder? }\end{array}$ & $\begin{array}{l}\text { Plastic and Reconstructive Surgery; } \\
\text { 129(2):406e-407e }\end{array}$ \\
\hline Picavet V. ${ }^{6}$ & Diciembre 2011 & $\begin{array}{l}\text { Screening tools for body dysmorphic } \\
\text { disorder in a cosmetic surgery setting }\end{array}$ & Laryngoscope; 121(12):2535-2541 \\
\hline Gilmartin J. ${ }^{15}$ & Julio 2011 & $\begin{array}{l}\text { Contemporary cosmetic surgery: the } \\
\text { potential risks and relevance for practice }\end{array}$ & $\begin{array}{l}\text { Journal of Clinical Nursing; } 20 \\
(13-14): 1801-1809\end{array}$ \\
\hline Gorney M. ${ }^{2}$ & Diciembre 2010 & $\begin{array}{l}\text { Recognition and management of the patient } \\
\text { unsuitable for aesthetic surgery }\end{array}$ & $\begin{array}{l}\text { Plastic and Reconstructive Surgery; } \\
\text { 126(6):2268-2271 }\end{array}$ \\
\hline Lai C.S. ${ }^{16}$ & Septiembre 2010 & $\begin{array}{l}\text { Body dysmorphic disorder in patients with } \\
\text { cosmetic surgery }\end{array}$ & $\begin{array}{l}\text { The Kaohsiung Journal of Medical } \\
\text { Science; 26(9):478-482 }\end{array}$ \\
\hline Conrado L.A. ${ }^{17}$ & Agosto 2010 & $\begin{array}{l}\text { Body dysmorphic disorder among } \\
\text { dermatologic patients: prevalence and } \\
\text { clinical features }\end{array}$ & $\begin{array}{l}\text { Journal of the American Academy of } \\
\text { Dermatology; 63(2):235-243 }\end{array}$ \\
\hline Calogero R.M..$^{18}$ & Enero 2010 & $\begin{array}{l}\text { Predicting excessive body image concerns } \\
\text { among British university students: the } \\
\text { unique role of Appearance-based Rejection } \\
\text { Sensitivity }\end{array}$ & Body Image; 7(1):78-81 \\
\hline
\end{tabular}

afectada su capacidad de reconocimiento de la enfermedad medida con la Brown Assesment of Beliefs Scale (BABS) (29). Estos pacientes afirman que los defectos corporales que perciben son reales, incluso cuando se les demuestra objetivamente que no lo son (30). Existe evidencia de que los pacientes con TDC que buscan ayuda con especialistas en salud mental presentan mejor nivel de conciencia de enfermedad que los que no lo hacen (31).

\section{Comorbilidades}

Tal como sucede en otras patologías psiquiátricas, el TDC suele asociarse a otras condiciones de salud mental. La prevalencia de trastornos de personalidad en pacientes diagnosticados con esta patología es de 47,9\% (10).

Phillips estudió 200 pacientes con TDC en los que encontró una mayor prevalencia de trastorno de personalidad paranoide $(10,9 \%)$, limítrofe $(11,8 \%)$, evitativa $(26,9 \%)$, obsesiva $(16,8 \%)$, dependiente $(5 \%)$ y antiso- 
Tabla II. Criterios Diagnósticos del DSM- IV TR para el diagnóstico del TDC

A. Preocupación por algún defecto imaginado del aspecto físico. Cuando hay leves anomalías físicas, la preocupación del individuo es excesiva

B. La preocupación provoca malestar clínicamente significativo o deterioro social, laboral o de otras áreas importantes de la actividad del individuo

C. La preocupación no se explica mejor por la presencia de otro trastorno mental (p.ej., insatisfacción con el tamaño y la silueta corporales en la anorexia nerviosa)

Tabla III. Adaptado de las recomendaciones preliminares para nuevos criterios diagnósticos del TDC en el DSM-V ${ }^{8}$

A. Preocupación por un defecto percibido en la apariencia física que no es observable o parece leve para los otros

B. La preocupación causa malestar clínico significativo (p. ej. ánimo depresivo, ansiedad, vergüenza) o desajuste social, ocupacional o en otras áreas importantes de funcionamiento (p.ej. escuela, relaciones, hogar)

C. Las preocupaciones por la apariencia no se restringen a preocupaciones por grasa corporal o peso en personas con desorden alimenticio

Especificar si la sospecha de TDC se caracteriza por :

- Buena conciencia de enfermedad (insight): reconoce que las creencias del TDC son definitivamente o probablemente falsas, o que pueden no ser verdaderas

- Poco conciencia de enfermedad (insight): piensa que las creencias del TDC son probablemente verdaderas

- Creencias delirantes con respecto a la apariencia: completamente convencido de que las creencias del TDC son verdaderas

Especificar si:

- Presencia de dismorfia muscular como forma del TDC (creencia de que un físico-culturista es muy pequeño o con musculatura insuficiente)

Tabla IV. Comorbilidades psiquiátricas del TDC

\begin{tabular}{|l|l|}
\hline Trastorno obsesivo-compulsivo & $8-37 \%$ \\
\hline Fobia social & $11-13 \%$ \\
\hline Tricotilomanía & $26 \%$ \\
\hline Trastorno depresivo mayor & $14-42 \%$ \\
\hline
\end{tabular}

cial $(6,7 \%)$. En cambio, otros trastornos de personalidad como el narcisista, el histriónico, el esquizoide o el esquizo típico no se vieron asociados con TDC (31). La relación con otras comorbilidades psiquiátricas se detalla en la Tabla IV $(10,32)$.
Tabla V. Partes del cuerpo de mayor preocupación (adaptado de Phillips et al.) ${ }^{18}$

\begin{tabular}{|l|l|}
\hline Piel & $80,0 \%$ \\
\hline Pelo & $57,5 \%$ \\
\hline Nariz & $39,0 \%$ \\
\hline Abdomen & $32,0 \%$ \\
\hline Dientes & $29,5 \%$ \\
\hline Peso & $29,0 \%$ \\
\hline Mamas & $26,0 \%$ \\
\hline Glúteos & $26,0 \%$ \\
\hline Ojos & $20,0 \%$ \\
\hline Cintura & $20,0 \%$ \\
\hline Cejas & $21,5 \%$ \\
\hline Apariencia facial general & $29,5 \%$ \\
\hline Hipotrofia muscular & $18,0 \%$ \\
\hline Piernas & $18,0 \%$ \\
\hline Mentón & $14,5 \%$ \\
\hline Labios & $14,5 \%$ \\
\hline Brazos & $13,5 \%$ \\
\hline Caderas & $10,5 \%$ \\
\hline Mejillas & $16,0 \%$ \\
\hline Orejas & $2 \%$ \\
\hline Tamaño / Forma facial & $2,5 \%$ \\
\hline
\end{tabular}

\section{Herencia}

En el mismo estudio de Phillips se establece que el $5,8 \%$ de los pacientes con TDC tienen un familiar en primer grado con la misma patología. El autor del artículo asegura que podría existir un componente genético aún no descubierto, pero que se requieren mayores estudios poblacionales para fortalecer dicha hipótesis (31).

\section{Partes del cuerpo más afectadas}

Las partes del cuerpo por las que con mayor frecuencia existe preocupación serían la piel, el pelo, la nariz, el abdomen, los dientes, el peso corporal y las mamas (Tabla V).

No hemos de olvidar que los pacientes que padecen TDC nos suelen percibir un defecto único, sino que pueden tener 5 a 7 disconformidades con diferentes partes de su cuerpo de forma simultánea (10).

\section{Cogniciones en el TDC}

Al explorar cuánto tiempo del día los pacientes con TDC se encuentran reflexionando sobre sus defectos, en- 
contramos que un $40 \%$ de ellos invierten hasta 3 a 8 horas diarias; un $25 \%$ lo hacen más de 8 horas al día (33). Estos pacientes suelen presentar pensamientos intrusivos, difíciles de resistir y controlar, asociados a egodistonía importante (34). El contenido de estos pensamientos se caracterizan por presentar ideas sobrevaloradas acerca del cuerpo y de cualquier defecto que éste pueda presentar (35)

\section{Ideación suicida}

Las tasas de ideación suicida, intentos suicidas y suicidios concretados, son más elevadas en el TDC que en la población general a nivel mundial (36). Un estudio prospectivo que siguió a 200 individuos diagnosticados con TDC de 1 a 4 años presentó que un 57,8\% de los sujetos presentaron ideación suicida (10-25 veces más que la población general de EE.UU.); que un 2,6\% presentó intentos suicidas durante el periodo de seguimiento, y que un $1,5 \%$ realizó intentos suicidas atribuidos directamente al TDC. Solo un 0,3\% completó el suicidio (37).

Sin embargo, esto podría deberse en parte a los factores de riesgo en común que presentan los pacientes que padecen esta enfermedad, y entre los cuales destaca un alta comorbilidad psiquiátrica, altos niveles de ansiedad, baja autoestima, hospitalizaciones repetidas y depresión (38).

\section{Satisfacción postquirúrgica}

Crerand publicó un estudio en el que incluyó a 200 individuos diagnosticados con TDC a los que se les habían realizado intervenciones de Cirugía Estética. Sólo un 3\% de ellos resultó beneficiado por el procedimiento quirúrgico que disminuyó su psicopatología inicial (39). La disconformidad con el aspecto físico persistió en la gran mayoría de los pacientes del grupo de estudio. Otro trabajo con una muestra de 250 pacientes en condiciones similares, señala que sólo el 7\% de los pacientes presentó un resultado postoperatorio satisfactorio (40).

Veale, en un estudio menor con 50 pacientes, afirma que un $81,0 \%$ de los pacientes con TDC que consultaron sobre Cirugía Estética no quedaron satisfechos con su cirugía. De estos casos, en 2 de cada 5 los cirujanos sufrieron amenazas por parte del paciente, física o legalmente (1). Incluso está descrito que la Cirugía Estética podría empeorar el cuadro de TDC, ya que el paciente tiene ideas sobrevaloradas y altas expectativas de resultado quirúrgico (41), si bien también hay un estudio que demuestra lo contrario, estableciendo que 83 de cada 100 pacientes demuestran mejoría tras la operación (42). No estaría por tanto del todo claro que la Cirugía Estética en este tipo de pacientes sea una contraindicación absoluta (41).

\section{Discusión}

Tras la revisión practicada podemos llegar a la conclusión de que la preocupación de los cirujanos plásticos por el TDC está completamente justificada. Efectivamente, existe una mayor prevalencia del trastorno en sus consultas que en las de otro tipo y la subdetección de estos pacientes a la hora del diagnóstico clínico es muy importante. Esto se debe a que el trastorno pasa fácilmente desapercibido cuando no se busca de forma dirigida.

La importancia de un diagnóstico certero está en que no solo permite excluir este tipo de pacientes de cirugías que pretendan corregir defectos imaginarios, sino que también permite derivar a un tratamiento adecuado los casos de TDC junto con sus posibles comorbilidades psiquiátricas.

Resulta muy interesante la caracterización que se ha podido llegar a hacer de estos pacientes en el contexto de la consulta de Cirugía Estética y Dermatológica, donde se concentran de forma mayoritaria los casos de TDC. También se ha podido reconocer que una mejor conciencia de enfermedad por parte de los pacientes se asocia a un mejor pronóstico, así como identificar las partes del cuerpo más frecuentemente implicadas.

Todo paciente en el que se sospeche TDC debe ser derivado a un psiquiatra, siendo el tratamiento de elección psicoterapia y farmacoterapia (43). El estrés que significa para estos pacientes el no ver una mejoría tras someterse a una cirugía puede empeorar la condición. La agresión verbal, física e incluso las acciones judiciales pueden evitarse de esta forma si logramos excluir los casos de afectación grave (44).

Por lo tanto, debemos tener mucho criterio en el momento de seleccionar a los pacientes candidatos para someterse a una intervención de Cirugía Estética. Es perentorio ampliar los estudios existentes sobre la materia, particularmente en nuestra región, para poder caracterizar mejor a nuestra población consultante y diseñar estrategias rápidas de cribado, fáciles de implementar y costoefectivas (6). Algunos autores han propuesto instrumentos para conseguir este fin $(13,45)$. Sin embargo, aún carecen de evidencia que los valide. Por ahora, se recomienda que el tema sea abordado en los cursos de formación para cirujanos plásticos. De la misma forma, es fundamental el contacto cercano de estos especialistas con psiquiatras calificados, de forma que llegado el caso, sea posible tratar a los pacientes con TDC desde una perspectiva integradora y multidisciplinaria.

\section{Conclusiones}

A modo de corolario podemos decir que el Trastorno Dismórfico Corporal es una condición psiquiátrica en la que el individuo se encuentra perturbado por una percepción anómala de un defecto corporal en su propio cuerpo. Su prevalencia es más alta entre pacientes que consultan por cirugías estéticas, ya que suelen buscar una solución quirúrgica para sus defectos imaginarios. Es de suma importancia poder diagnosticar esta patología para 
que tras ser evaluados por un psiquiatra, estos pacientes puedan recibir el tratamiento adecuado.

La cirugía tiene un efecto limitado sobre sus percepciones, por lo que se debe juzgar apropiadamente antes de operar a un paciente en el que se sospeche TDC. Excluir a un paciente de una alternativa quirúrgica es una decisión compleja, pero puede ser una conducta beneficiosa para el paciente y para el propio cirujano.

Aún necesitamos más estudios en el contexto iberolatinoamericano para conocer la dimensión local del problema y poder así desarrollar instrumentos que faciliten la detección del TDC en el contexto de la consulta especializada de Cirugía Estética.

\section{Dirección del autor}

Dr. Gunther Rochefort Ciscutti

Lo Fontecilla 101, Oficina 413

Santiago, Chile

e-mail: grochefort@gmail.com

\section{Bibliografía}

1. Veale D, Boocock A, Gournay K, et al.: Body dysmorphic disorder. A survey of fifty cases. Br J Psychiatry 1996; 169: 196-201.

2. Gorney M.: Recognition and management of the patient unsuitable for aesthetic surgery. Plast Reconstr Surg 2010; 126: 2268-2271.

3. Association AP. Diagnostic and Statistical Manual of Mental Disorders, 4th Edition, Text Revision (DSM-IVTR). American Psychiatric Association, 2000.

4. Morselli E.: Sulla dismorfofobia e sulla tafefobia, due forme non ancora descritte di Passia con idee fisse. $\mathrm{Bo}$ lletino della Regia Accad delle Sci Mediche di Genova 1891; VI: 110-119.

5. Association AP. Diagnostic and statistical manual of mental disorders, 3rd Edition rev. (DSM-III-R). Washington, DC., 1987.

6. Picavet V, Gabriëls L, Jorissen M, Hellings PW.: Screening tools for body dysmorphic disorder in a cosmetic surgery setting. Laryngoscope 2011; 121: 2535-2541.

7. Veale D, Eshkevari E, Ellison N, Cardozo L, Robinson D, Kavouni A.: Validation of genital appearance satisfaction scale and the cosmetic procedure screening scale for women seeking labiaplasty. J Psychosom Obstet Gynaecol 2013; 34: 46-52.

8. Gupta MA, Gupta AK.: Evaluation of cutaneous body image dissatisfaction in the dermatology patient. Clin Dermatol; 31: 72-79.

9. Morselli PG, Boriani F.: Should plastic surgeons operate on patients diagnosed with body dysmorphic disorders? Plast Reconstr Surg 2012; 130: 620e-622e; author reply $622 \mathrm{e}$.

10. Ericksen WL, Billick SB.: Psychiatric issues in cosmetic plastic surgery. Psychiatr $Q 2012 ; 83: 343-352$.

11. Kyle A.: Body dysmorphia and plastic surgery. Plast Surg Nurs; 32: 96-98; Quiz 99-100.

12. Clarke A, Hansen ELE, White P, Butler PEM.: Low priority? A cross sectional study of appearance anxiety in 500 consecutive referrals for cosmetic surgery. Psychol Health Med 2012; 17: 440-446.
13. Mulkens S, Bos AER, Uleman R, Muris P, Mayer B, Velthuis P.: Psychopathology symptoms in a sample of female cosmetic surgery patients. J Plast Reconstr Aesthet Surg 2012; 65: 321-327.

14. De Brito MJA, Nahas FX, Ferreira LM.: Should plastic surgeons operate on patients diagnosed with body dysmorphic disorder? Plast Reconstr Surg 2012; 129: 406e-407e.

15. Gilmartin J.: Contemporary cosmetic surgery: the potential risks and relevance for practice. J Clin Nurs 2011; 20: 1801-1809.

16. Lai C-S, Lee S-S, Yeh Y-C, Chen C-S.: Body dysmorphic disorder in patients with cosmetic surgery. Kaohsiung J Med Sci 2010; 26: 478-482.

17. Conrado LA, Hounie AG, Diniz JB, et al.: Body dysmorphic disorder among dermatologic patients: Prevalence and clinical features. J Am Acad Dermatol 2010; 63: 235-243.

18. Calogero RM, Park LE, Rahemtulla ZK, Williams KCD.: Predicting excessive body image concerns among British university students: the unique role of Appearance-based Rejection Sensitivity. Body Image 2010; 7: 78-81.

19. World Health Organization. ICD-10 Classification of Mental and Behavioural Disorders; Diagnostic Criteria for Research. Geneva, 1992.

20. Phillips KA, Wilhelm S, Koran LM, et al.: Body dysmorphic disorder: some key issues for DSM-V. Depress Anxiety 2010; 27: 573-591.

21. Koran LM, Abujaoude E, Large MD, Serpe RT.: The prevalence of body dysmorphic disorder in the United States adult population. CNS Spectr 2008; 13: 316-322.

22. Faravelli C, Salvatori S, Galassi F, Aiazzi L, Drei C, Cabras P.: Epidemiology of somatoform disorders: a community survey in Florence. Soc Psychiatry Psychiatr Epidemiol 1997; 32: 24-29.

23. Biby EL.: The relationship between body dysmorphic disorder and depression, self-esteem, somatization, and obsessive-compulsive disorder. J Clin Psychol 1998; 54: 489-499.

24. Borda T, Neziroglu F, Santos N, Donnelly K, Rivera RP.: Status of body dysmorphic disorder in Argentina. $J$ Anxiety Disord 2011; 25: 507-512.

25. Phillips KA.: Somatoform and factitious disorders. Washington, DC., American Psychiatric Publishing, 2001.

26. Ishigooka J, Iwao M, Suzuki M, Fukuyama Y, Murasaki M, Miura S.: Demographic features of patients seeking cosmetic surgery. Psychiatry Clin Neurosci 1998; 52: 283-287.

27. Aouizerate B, Pujol H, Grabot D, et al.: Body dysmorphic disorder in a sample of cosmetic surgery applicants. Eur Psychiatry 2003; 18: 365-368.

28 Rief W, Buhlmann U, Wilhelm S, Borkenhagen A, Brähler E.: The prevalence of body dysmorphic disorder: a population-based survey. Psychol Med 2006; 36: 877-885.

29 Eisen JL, Phillips KA, Baer L, Beer DA, Atala KD, Rasmussen SA.: The Brown Assessment of Beliefs Scale: reliability and validity. Am J Psychiatry 1998; 155: 102-108.

30 Phillips KA, Menard W, Pagano ME, Fay C, Stout RL.: Delusional versus nondelusional body dysmorphic disorder: clinical features and course of illness. $J$ Psychiatr Res 2006; 40: 95-104.

31 Phillips K a, Menard W, Fay C, Weisberg R.: Demographic characteristics, phenomenology, comorbidity, 
and family history in 200 individuals with body dysmorphic disorder. Psychosomatics 2005; 46: 317-325.

32. Hollander E, Cohen LJ, Simeon D.: Body dysmorphic disorder. Psychiatr Ann 1993; 23: 359-364.

33. Phillips KA.: The Broken Mirror: Understanding and Treating Body Dysmorphic Disorder. Oxford University Press, 2005.

34. Phillips KA.: Understanding Body Dysmorphic Disorder. Oxford University Press, 2009.

35. Neziroglu F, Stevens KP, McKay D, Yaryura-Tobias JA.: Predictive validity of the overvalued ideas scale: outcome in obsessive-compulsive and body dysmorphic disorders. Behav Res Ther 2001; 39: 745-756.

36. WHO I Suicide prevention (SUPRE). Disponible en: http://www.who.int/mental_health/prevention/suicide/sui cideprevent/en/

37. Phillips KA, Menard W.: Suicidality in body dysmorphic disorder: a prospective study. Am J Psychiatry 2006; 163: 1280-1282.

38. Phillips KA, Coles ME, Menard W, Yen S, Fay C, Weisberg RB.: Suicidal ideation and suicide attempts in body dysmorphic disorder. J Clin Psychiatry 2005; 66: 717-725.
39. Crerand CE, Phillips KA, Menard W, Fay C.: Nonpsychiatric medical treatment of body dysmorphic disorder. Psychosomatics; 46: 549-555.

40. Phillips KA, Grant J, Siniscalchi J, Albertini RS.: Surgical and nonpsychiatric medical treatment of patients with body dysmorphic disorder. Psychosomatics 2001; 42: $504-510$

41. Shridharani SM, Magarakis M, Manson PN, Rodriguez ED.: Psychology of plastic and reconstructive surgery: a systematic clinical review. Plast Reconstr Surg 2010; 126: 2243-2251.

42. Edgerton MT, Langman MW, Pruzinsky T.: Plastic surgery and psychotherapy in the treatment of 100 psychologically disturbed patients. Plast Reconstr Surg 1991; 88: 594-608.

43. Phillips KA.: Body dysmorphic disorder and depression: theoretical considerations and treatment strategies. Psychiatr Q 1999; 70: 313-331.

44. Grossbart T a, Sarwer DB.: Psychosocial issues and their relevance to the cosmetic surgery patient. Semin Cutan Med Surg 2003; 22: 136-147.

45. Blackburn VF, Blackburn a V.: Taking a history in aesthetic surgery: SAGA--the surgeon's tool for patient selection. J Plast Reconstr Aesthet Surg 2008; 61: 723-729. 